\title{
Percepción de la calidad de vida y satisfacción del paciente en cuidados paliativos
}

\author{
Perception of quality of life and patient satisfaction in palliative care \\ Jesús Melchor Santos-Flores,* Eddy García-Zapatał
}

\section{RESUMEN}

Introducción: La atención al paciente en fase terminal es un importante problema de salud que se encuentra en aumento por el incremento en la prevalencia de las enfermedades crónicas y el envejecimiento, de edad avanzada, con dependencia, comorbilidad y fragilidad, con necesidades de atención y como más importante la atención en cuidados paliativos. En la actualidad, se ha propuesto como evaluación del cuidado la satisfacción y la calidad de vida. Objetivo: Determinar la relación entre el nivel de calidad de vida y la satisfacción del paciente en el cuidado paliativo. Material y métodos: El presente estudio es descriptivo-correlacional. La muestra de interés estuvo conformada por 60 pacientes paliativos, el muestreo fue no probabilístico tipo censal, seleccionando a toda la población de pacientes paliativos. Resultados: La media de edad fue 70.3 años, el sexo femenino predominó con 51.7\%, 33.3\% presentó cáncer. La mayoría reportó dolor moderado (35\%). Se encontró una media de 62.8 (DE = 24.98) de calidad de vida. En satisfacción en cuidados paliativos con M = 6.1 (DE = 0.97). Se correlacionó positiva y significativamente la calidad de vida y satisfacción en cuidados paliativos ( $\mathrm{rs}$ = $0.31, p=0.01$ ). Conclusión: La calidad de vida y la satisfacción del paciente en cuidados paliativos son fundamentales en el contexto de la evaluación de los resultados. Se encontró una moderada relación entre la calidad de vida y la satisfacción del paciente, así como puntuaciones altas en síntomas: constipación, náuseas y disnea.

Palabras clave: Cuidados paliativos, satisfacción del paciente, calidad de vida, psicología.

\begin{abstract}
Introduction: Attention to patients in the terminal phase is an important health problem that is on the rise, due to the increase in the prevalence of chronic diseases and aging, of advanced age, with dependence, comorbidity and frailty, with care needs and as more important the attention in palliative care. At present, satisfaction and quality of life have been proposed as an assessment of care. Objective: To determine the relationship between the level of quality of life and patient satisfaction in palliative care. Material and methods: The present study is descriptive-correlational. The sample of interest consisted of 60 palliative patients; the sampling was non-probabilistic, census type, selecting the entire population of palliative patients. Results: The mean age was 70.3 years; the female sex predominated with 51.7\%, 33.3\% presented Cancer. Most reported moderate pain (35\%). A mean of 62.8 (SD $=24.980)$ of Quality of Life was found. In Palliative Care Satisfaction of 6.1 (SD = 0.971). Quality of Life and Satisfaction in palliative care were positively and significantly correlated ( $r s=0.314, p=0.015)$. Conclusion: The quality of life and patient satisfaction in palliative care are fundamental in the context of the evaluation of the results. A moderate relationship was found between quality of life and patient satisfaction, as well as high scores in symptoms: constipation, nausea and dysfunction.
\end{abstract}

Keywords: Palliative care, patient satisfaction, quality of life, psychology.

* Secretaría de Salud. Hospital General Sabinas Hidalgo «Virginia Ayala de Garza». Maestría en Ciencias de Enfermería.

* Secretaría de Salud. Jurisdicción Sanitaria No. 5. Maestría en Administración y Gestión de los Servicios de Salud.

Correspondencia: JMSF, santos_meme_10@ hotmail.com Conflicto de intereses: Ninguno.

Citar como: Santos-Flores JM, García-Zapata E. Percepción de la calidad de vida y satisfacción del paciente en cuidados paliativos. Rev CONAMED. 2021; 26(3): 109-115. https:// dx.doi.org/10.35366/101675 Financiamiento: Ninguno.

Recibido: 28/06/2021

Aceptado: 13/09/2021 


\section{INTRODUCCIÓN}

La atención al paciente en fase terminal es un importante problema de salud que se encuentra en aumento, una de las causas es el incremento en la prevalencia de las enfermedades crónicas (EC) y el envejecimiento. ${ }^{1,2}$ Los pacientes con EC son complejos, generalmente de edad avanzada, con gran dependencia, comorbilidad y fragilidad, con necesidades de atención diferentes y una de las más importantes es la atención en cuidados paliativos (CP)!

De acuerdo con la Organización Mundial de la Salud $(\mathrm{OMS})^{3}$ alrededor de 40 millones de personas necesitan CP; sin embargo, a nivel mundial, sólo $14 \%$ de los que necesitan CP lo reciben. La necesidad de los CP seguirá en aumento como consecuencia de la creciente carga de las EC y del proceso de envejecimiento de la población. ${ }^{3}$ Existen distintas enfermedades que requieren $C P$, la mayoría clasificadas como EC: enfermedades cardiovasculares (38.5\%), cáncer (34\%), enfermedades respiratorias crónicas (10.3\%), SIDA (5.7\%) y diabetes (4.6\%).

Según la $\mathrm{OMS}^{4}$ es una parte esencial en la lucha contra las enfermedades terminales y pueden otorgarse de forma sencilla y económica, hacen referencia a la mejora de la calidad de vida (CV) de los pacientes y las familias. Los objetivos de los CP son el control de síntomas como el dolor, la atención de los problemas psicológicos, sociales y espirituales, dirigidos al alivio del sufrimiento de la persona enferma incluyendo a su familia. ${ }^{5}$ Por lo tanto, la esencia del CP es el alivio del sufrimiento de la persona enferma incluyendo a su familia. ${ }^{6}$

En la actualidad, se ha propuesto como evaluación del cuidado otorgado por parte del personal de salud la evaluación de la satisfacción del paciente en el cuidado paliativo (SPCP), siendo un concepto próximo a la CV.7-9

Al ser un concepto multidimensional, la CV incluye el componente físico, caracterizado por la ausencia de dolor, dificultad respiratoria, síntomas digestivos y otros causantes de molestias; también incluye un componente psicológico, determinado por la ausencia de emociones negativas, ansiedad y depresión, y un proceso de adaptación adecuado a la enfermedad; asimismo, un componente social, posibilidad de interacción con otros individuos sin alteración en la comunicación y un componente espiritual en el que se percibe una autoestima válida en relación con el conocimiento y la percepción del control de la propia vida. ${ }^{10}$

Cada componente de la CV del paciente en CP supone el resultado del personal de salud de los servicios de atención, desempeñando un papel fundamental en la mejora de la CV en los CP, es por ello que su evaluación constituye uno de los objetivos primordiales para ofrecer una atención de calidad. 10,17 La CV percibida por un paciente que requiere de CP está fuertemente modulado por procesos vinculados a la evaluación que el individuo realiza tanto de sus condiciones objetivas de vida como del grado de satisfacción, ${ }^{12}$ otros autores sostienen que la CV se mide en términos de utilidad o satisfacción de necesidades..$^{13}$

De este modo, otro concepto que ha tomado interés ha sido la satisfacción del paciente, siendo un concepto que se ha sido utilizado para valorar la calidad de la atención. En la actualidad, es uno de los métodos más aceptados, su objetivo es identificar los aspectos del cuidado paliativo que son susceptibles de mejora. ${ }^{8}$ Durante la fase final de la vida, la SPCP puede ser una de las mejores opciones cuando se trata de evaluar indicadores de calidad; 8 según algunos autores se ha relacionado con la CV, 9,10 otros autores no han encontrado relación significativa. ${ }^{7,14}$ Por lo anterior, el objetivo del presente estudio fue analizar la correlación entre la percepción de la calidad de vida y la satisfacción del paciente en el cuidado paliativo.

\section{MATERIAL Y MÉTODOS}

\section{Diseño}

El presente estudio es cuantitativo de tipo descriptivo transversal.

\section{Población y muestra}

La población de interés estuvo conformada por 60 pacientes paliativos ubicados en municipios al norte del estado de Nuevo León. El muestreo fue no probabilístico tipo censal, seleccionando a toda la población de pacientes paliativos a cargo de la Jurisdicción No. 5 ubicada en Sabinas Hidalgo, Nuevo León. 


\section{Instrumentos de medición}

Para el presente estudio se aplicaron la cédula de datos sociodemográficos y clínicos, y tres instrumentos para medir las variables principales del estudio: la European Organisation for Research and Treatment of Cancer (EORTC) en su versión Quality of Life core Questionnaire-core Palliative de 15 ítems (QLQC15-PAL), la versión In-Patient Satisfaction with Care Questionnaire de 32 items (IN-PATSAT32) y la encuesta Evaluación de la Satisfacción de los Usuarios en Cuidados Paliativos (ESUCP).

Para el registro de las variables sociodemográficas y clínicas se empleó la Cédula de Datos Sociodemográficos y Clínicos (CDSyC). Para medir la CV en los CP se utilizó la escala EORTC QLQ-C15PAL de Groenvold y su equipo, ${ }^{15}$ en su versión al español validada por Arraras y colaboradores, ${ }^{16}$ cuenta con 15 ítems e incluye subescalas funcionales (física y emocional), de calidad de vida global y sintomática, esta última para evaluación de síntomas como fatiga, náuseas/vómitos, dolor, insomnio, disnea, pérdida de apetito y estreñimiento. Las preguntas se miden a través de la escala tipo Likert según si la situación se presenta en absoluto = 1; un poco = 2; bastante $=3$; o mucho $=4$.

La escala de síntomas se mide en rangos de 0 a 100 , siendo $0=$ muy buen control y 100 = pésimo control. Las subescalas funcionales pueden ser medidas a través de la sumatoria de algunas preguntas: las preguntas 1, 2 y 3 evalúan la capacidad funcional física; las preguntas 13 y 14, la capacidad funcional emocional en rangos de $\mathrm{O}$ a 100 , siendo $\mathrm{O}=$ muy mal funcionamiento y 100 = buen funcionamiento. La pregunta 15 determina la autopercepción de la CV del paciente y tiene siete niveles, siendo 1 = pésima y 7 = excelente. De acuerdo con la literatura, los valores de Alfa de Cronbach oscilan entre 0.70 a 0.90, 7,17 los cuales se consideran aceptables y buenos.

Para evaluar la SPCP se utilizó la escala EORTC IN-PATSAT32 de Bredart y sus colegas, ${ }^{18}$ en su versión al español validada por Arraras y su equipo, ${ }^{16}$ cuenta con 32 ítems y evalúa los aspectos multidimensionales de la satisfacción de la atención. Los ítems son agrupados en 14 subescalas: calidad de los servicios médicos (habilidades técnicas, habilidades interpersonales, provisión de la información y disponibilidad), de los cuidados de enfermería (habilidades técnicas, habilidades interpersonales, provisión de la información y disponibilidad), calidad administrativa (información, amabilidad, tiempo de espera, acceso, confort y limpieza) y el último ítem sobre satisfacción en general. Cada uno de los ítems se puntúa en una escala tipo Likert de cinco puntos: 1 = mal, 2 = regular, 3 = bien, 4 = muy bien y 5 = excelente. Todas las puntuaciones se transforman a una escala de 0 a 100 puntos, en la que las puntuaciones más elevadas indican niveles mayores de satisfacción. De acuerdo con la literatura, los valores de Alfa de Cronbach oscilan entre 0.77 a 0.97,16,18 los cuales se consideran aceptables y buenos.

Además, se utilizó el Área Psicológica-Apoyo Emocional de la Encuesta de ESUCP, recopila información fundamental sobre la atención en los CP, atención integral, necesidades físicas, psicológicas, sociales y espirituales. Está compuesta por 23 ítems, agrupados en cinco áreas: física (preguntas 1, 2 y 5), psicológica (6A, 6B, 7A, 7B y 13), espiritual (8A, 8B, 8C, 9A, 9B), social (14, 15, 16 y 17) y comunicación (10, 11 y 12), el ítem 18 no se agrupa. ${ }^{2}$ Sin embargo, se utilizaron sólo los ítems del área psicológica correspondientes a las preguntas 7A (apoyo emocional paciente evolución), 7B (apoyo emocional paciente últimos momentos), 13 (momento facilitada ayuda emocional); éstos presentan una escala tipo Likert: siempre $=4$, a menudo $=3$, pocas veces $=2$, nunca = 1; cuenta con una valoración global de uno a cuatro, donde 1 y 2 = apoyo emocional no satisfecho, 4 y 5 = apoyo emocional satisfecho. La encuesta ESUCP cuenta con un Alfa de Cronbach de 0.95, en cuanto al área psicológica presenta un Alfa de Cronbach de 0.91.2

\section{Análisis de datos}

Los datos se capturaron y analizaron mediante el uso del paquete estadístico Statistical Package for the Social Sciences (SPSS) versión 22 para Windows 10. Se realizó análisis descriptivo a través de frecuencias, porcentajes, medidas de tendencia central y variabilidad. Se utilizó estadística no paramétrica, $\chi^{2}$ de Pearson para variables categóricas, la prueba $U$ de Mann-Whitney aplicada a dos muestras independientes, así como la prueba $\mathrm{H}$ de Kruskal-Wallis aplicada a tres muestras independientes y el coeficiente de correlación de 
Spearman. Se utilizó un nivel de significancia del 95\% como valor de referencia $p<0.05$.

\section{Consideraciones éticas}

El estudio se apegó a lo dispuesto en el reglamento de la Ley General de Salud en Materia de Investigación para la Salud, en su última reforma publicada DOF 02-04-2014. ${ }^{19}$

\section{RESULTADOS}

La media de edad reportada fue de 70.3 años $(\mathrm{DE}=17.712$, rango intercuartílico $=61-81)$, con una media de años de estudio de 7.7 (DE $=4.153$, rango intercuartílico $=6-9$ ); la media de años con la enfermedad fue de 2.65 (DE $=4.743$, rango intercuartílico $=1-3$ ). En la Tabla 7 se presentan las frecuencias y porcentajes en las características sociodemográficas y clínicas de la muestra de estudio. El sexo femenino predominó con 51.7\%, el estado civil más prevalente fue casado (38.3\%). En cuanto a las características clínicas, 33.3\% presentó como diagnóstico médico cáncer (mama, próstata, óseo, cervicouterino, renal, páncreas y estómago), en 10\% evento cerebrovascular, en 8.3\% insuficiencia cardiaca congestiva y enfermedad pulmonar obstructiva crónica. De acuerdo con el nivel del dolor la mayoría reportó un dolor moderado de cuatro a seis puntos (35\%).

De acuerdo con la calidad de vida de la muestra de estudio (síntomas: 0 = muy buen control y 100 = pésimo control), el síntoma menos controlado fue la constipación con una media de 58.7 (DE = 33.135, rango intercuartílico = 50-75), el síntoma mejor controlado fue la función física $(M=32.6$, $\mathrm{DE}=29.284$, rango intercuartílico $=25-75)$ y la fatiga ( $M=38.9$, DE = 31.897, rango intercuartílico = 0-59.3); sin embargo, todos los síntomas en general estuvieron regularmente controlados. De acuerdo con la calidad de vida global se encontró una media de 62.8 (DE = 24.980, rango intercuartílico = 42.8-85.7), equivalente a 4.4 ( 1 = pésima $\mathrm{y}$ 7 = excelente), representando una media entre mala y regular (Tab/a 2).

La muestra obtuvo una media de satisfacción de 6.1 (DE = 0.971) (1 = pésima y 7 = excelente). Tuvo una satisfacción media de 83 (DE = 12.163, rango intercuartílico $=81.6-90.6)$ con los servicios
Tabla 1: Características sociodemográficas y clínicas de la muestra. $\mathrm{N}=60$.

\begin{tabular}{|c|c|}
\hline & $n(\%)$ \\
\hline \multicolumn{2}{|l|}{ Sexo } \\
\hline Masculino & $29(48.3)$ \\
\hline Femenino & $31(51.7)$ \\
\hline \multicolumn{2}{|l|}{ Estado civil de la muestra } \\
\hline Viudo & 17 (28.3) \\
\hline Soltero & $14(23.3)$ \\
\hline Casado & $23(38.3)$ \\
\hline Divorciado & $3(5.0)$ \\
\hline Unión libre & $3(5.0)$ \\
\hline \multicolumn{2}{|l|}{ Religión } \\
\hline Católica & $46(76.7)$ \\
\hline Cristiana & 8 (13.3) \\
\hline Testigo de Jehová & $4(6.7)$ \\
\hline Evangélica & $1(1.7)$ \\
\hline Ateo & $1(1.7)$ \\
\hline \multicolumn{2}{|l|}{ Persona principal de apoyo } \\
\hline Hijos & $32(53.4)$ \\
\hline Cuidador & $13(21.7)$ \\
\hline Esposos & $8(13.3)$ \\
\hline Padres & $2(3.4)$ \\
\hline Nietos & $1(1.7)$ \\
\hline Sobrinos & $1(1.7)$ \\
\hline Hermanos & $3(5.0)$ \\
\hline \multicolumn{2}{|l|}{ Características clínicas } \\
\hline \multicolumn{2}{|l|}{ Diagnóstico médico } \\
\hline Cáncer & $20(33.3)$ \\
\hline Evento cerebrovascular & $6(10.0)$ \\
\hline Insuficiencia cardiaca congestiva & $5(8.3)$ \\
\hline EPOC & $5(8.3)$ \\
\hline Demencia & $3(5.0)$ \\
\hline Oclusión intestinal & $3(5.0)$ \\
\hline Sepsis & $2(3.3)$ \\
\hline Traumatismo craneoencefálico & $2(3.3)$ \\
\hline Tumor & $2(3.3)$ \\
\hline Otros (gastrostomía, encefalopatía, & $12(20.4)$ \\
\hline pulmonar, atrofia muscular, urosepsis, & \\
\hline parálisis cerebral, isquemia cerebral, & \\
\hline angina de pecho inestable, depresión, & \\
\hline carcinoma, fibrosis quística) & \\
\hline \multicolumn{2}{|l|}{ Nivel de dolor } \\
\hline Sin dolor (0) & $4(6.7)$ \\
\hline Dolor leve (2) & $4(6.7)$ \\
\hline Dolor moderado (4-6) & $21(35.0)$ \\
\hline Dolor intenso (8) & $18(30.0)$ \\
\hline Máximo dolor inimaginable (10) & $12(20.0)$ \\
\hline
\end{tabular}


médicos, una media de 84.2 (DE = 12.852, rango intercuartílico = 79.1-94.7) con los servicios de enfermería y una media de 82.5 (DE = 15.232, rango intercuartílico = 72.8-96.1) con la organización que brinda los servicios (Tabla 3).

\begin{tabular}{lcr}
\multicolumn{2}{c}{$\begin{array}{c}\text { Tabla 2: Estadística descriptiva de la calidad } \\
\text { de vida en la muestra de estudio. } \mathrm{N}=60 .\end{array}$} \\
\hline QLQ-C15-PAL & \multicolumn{1}{c}{ RIC } \\
\hline Disnea & $49.1 \pm 37.666$ & $0-75.0$ \\
Dolor & $42.0 \pm 30.004$ & $25-62.5$ \\
Insomnio & $44.1 \pm 34.862$ & $0-75.0$ \\
Fatiga & $38.9 \pm 31.897$ & $0-59.3$ \\
Apetito & $47.0 \pm 37.135$ & $0-75.0$ \\
Náusea & $52.9 \pm 41.704$ & $0-75.0$ \\
Constipación & $58.7 \pm 33.135$ & $50-75.0$ \\
Función física & $32.6 \pm 29.284$ & $33.3-56.2$ \\
Función emocional & $48.5 \pm 34.757$ & $25-75.0$ \\
\hline
\end{tabular}

$\mathrm{RIC}=$ rango intercuartílico.
De acuerdo con la satisfacción del paciente en cuidados paliativos se encontró una media global de 88.3 (DE = 12.748, rango intercuartílico = 85.7100), equivalente a 6.1 (1 = pésima y 7 = excelente), representando una media entre buena y muy buena satisfacción.

Se reportaron altos porcentajes en cuanto a la frecuencia de apoyo emocional al paciente en cuidados paliativos durante la evolución de su enfermedad «a menudo» con $40 \%$ y «siempre» con $38.3 \%$, y durante los últimos momentos «a menudo» con 31.7\% y «siempre» con 41.7\% (Tabla 4).

No se reportaron diferencias significativas entre la CV y las variables sociodemográficas y clínicas como lo son el sexo, edad, escolaridad y nivel de dolor ( $p>0.05)$.

No se identificaron diferencias significativas entre la SPCP y las variables sociodemográficas y clínicas como el sexo, escolaridad y nivel de dolor ( $p>0.05)$; sin embargo, se encontraron diferencias significativas entre la SPCP y la edad, la media más alta se presentó en pacientes de entre 80 y 101 años de edad ( $M=94.9)$, en pacientes más jóvenes se

Tabla 3: Estadística descriptiva de la satisfacción del paciente en cuidados paliativos en la muestra de estudio. $\mathrm{N}=60$.

IN-PATSAT32

$\mathrm{RIC}$

\section{Servicios médicos}

Habilidades técnicas $(1,2,3)$

Habilidades interpersonales $(4,5,6)$

Provisión de la información $(7,8,9)$

Disponibilidad $(10,11)$

\section{Satisfacción con los cuidados de enfermería}

Habilidades técnicas $(12,13,14)$

Habilidades interpersonales $(15,16,17)$

Provisión de la información $(18,19,20)$

Disponibilidad $(21,22)$

\section{Satisfacción con la organización de los servicios}

Información $(23,25,26)$

Amabilidad (24)

Tiempo de espera $(27,28)$

Acceso $(29,30)$

Confort y limpieza (31)

$\begin{array}{crr} & 83.4 \pm 11.804 & 80-93.3 \\ & 84.6 \pm 13.739 & 80-93.3 \\ & 84.3 \pm 13.266 & 80-98.3 \\ \text { Promedio } & 77.3 \pm 17.356 & 80-90.0 \\ & 83.0 \pm 12.163 & 81.6-90.6\end{array}$

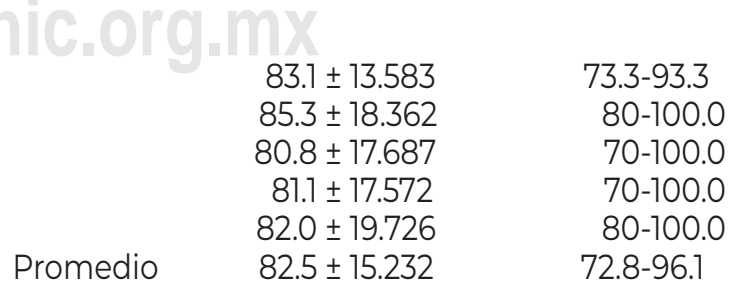

$\mathrm{RIC}=$ rango intercuartílico. 
presentaron medias más bajas de SPCP ( $M=84.7)$ $(H=7.667, p=0.022)$.

Se realizó la prueba de coeficiente de correlación de Spearman. Las variables CV y SPCP se correlacionaron de manera positiva y significativa ( $r s=0.314, p=0.015)$, lo que indica que a mayor $\mathrm{CV}$ mayor es la SPCP.

Entre la edad y el nivel del dolor se encontró correlación negativa y significativa ( $r s=-0.366, p=$ $0.004)$, lo que indica que a mayor edad menor dolor. Con respecto a la edad y a la SPCP se encontró relación positiva ( $r s=0.288, p=0.026$ ), lo que indica que a mayor edad mayor satisfacción.

\section{DISCUSIÓN}

La muestra total la constituyeron 60 pacientes en cuidados paliativos de un hospital de segundo nivel, valor que puede ser considerado aceptable teniendo en cuenta otros estudios consultados y las dificultades clínicas de esta población con respecto a la gravedad de los síntomas, la vulnerabilidad emocional y la frecuente fluctuación del estado de conciencia de origen orgánico. ${ }^{9}$

La edad promedio fue de 70.3 años, fue similar a la reportada por Hernández y su equipo 7 y Velilla y Jaramillo ${ }^{9}$ de 64 a 67 años, respectivamente. Con respecto al estado civil y la escolaridad más frecuentemente alcanzada, los hallazgos fueron similares a los encontrados por Gómez y sus colegas $^{20}$ y Velilla y Jaramillo. ${ }^{9}$ Fueron más frecuentes los pacientes casados y con estudios de grado secundario. No se encontró ningún estudio similar que midiera la religión, la procedencia ni el estrato socioeconómico. Sin embargo, algunos estudios consideran la religiosidad, el bienestar espiritual y la esperanza como determinantes importantes en la calidad de vida de los pacientes. ${ }^{9}$

Se encontró que la mayoría de los pacientes reportaron una calificación de la intensidad del dolor como moderado, otros autores encontraron en sus pacientes una intensidad del dolor leve; no obstante, los pacientes de otros estudios fueron incluidos desde el Servicio de Urgencias del Centro Hospitalario y muchos ya habían iniciado manejo analgésico, bien sea por el médico de urgencias o por cuidados paliativos. ${ }^{9}$ La calidad de vida global encontrada en nuestro estudio fue similar a la

\begin{tabular}{|c|c|c|}
\hline \multicolumn{3}{|c|}{$\begin{array}{l}\text { Tabla 4: Estadística descriptiva de la satisfacción } \\
\text { del paciente en cuidados paliativos-apoyo } \\
\text { emocional en la muestra de estudio. }\end{array}$} \\
\hline \multirow[b]{2}{*}{ ESUCP } & \multicolumn{2}{|c|}{ Apoyo emocional } \\
\hline & $\begin{array}{l}\text { Durante la } \\
\text { evolución, } \\
(\mathrm{N}=60) \\
\mathrm{n}(\%)\end{array}$ & $\begin{array}{c}\text { Durante } \\
\text { los últimos } \\
\text { momentos } \\
\text { (N = 59), } \\
\mathrm{n}(\%)\end{array}$ \\
\hline Nunca & $6(10.0)$ & $6(10.0)$ \\
\hline Pocas veces & 7 (11.7) & $9(15.0)$ \\
\hline A menudo & $24(40.0)$ & 19 (31.7) \\
\hline Siempre & $23(38.3)$ & 25 (41.7) \\
\hline
\end{tabular}

reportada por Hernández y colaboradores ${ }^{7}$ (51.7) y por Velilla y Jaramillo9 (49) a través del uso de la escala QLQ-C15-PAL.

En cuanto a la satisfacción del paciente, en el presente estudio se presentaron resultados altos de las subescalas > 80, similar a lo encontrado por Geidy. ${ }^{21}$ La puntuación más alta de las subescalas de satisfacción podría ser explicada no sólo por diferencias culturales en la población latinoamericana versus europea, sino también por el resultado del compromiso con el bienestar de los pacientes y la humanización de la atención. Se encontró una tendencia a la mejora en la calidad de vida con mayores puntajes para la satisfacción con los servicios de enfermería y la satisfacción general, similar a lo reportado por otros autores. ${ }^{9}$

\section{CONCLUSIÓN}

La calidad de vida y la satisfacción del paciente en cuidados paliativos son fundamentales en el contexto de la evaluación de los resultados. Se encontró una relación positiva de mediana intensidad entre la calidad de vida y la satisfacción del paciente, así como en puntuaciones altas en las subescalas de síntomas como constipación, náuseas y disnea. En cuanto a la satisfacción, se encuentran satisfechos con los cuidados de enfermería, el servicio médico y la organización de los servicios en general. 


\section{AGRADECIMIENTOS}

A la Lic. en Enfermería Francisca Julieta Valdez Ramírez, al Lic. Martín Zavala Moreno, Lic. en Enfermería Hermila Ruíz Martell, Lic. en Enfermería María Guadalupe Pérez Rodríguez y a la Lic. en Trabajo Social Sanjuana Martínez Cantú, por su compromiso y dedicación en la participación para la elaboración de esta investigación y por cada una de sus valiosas aportaciones para el mejoramiento de este trabajo.

\section{REFERENCIAS}

1. Ferrer PA, Lorenzo CD, González JMC, Verde IC, Baltanás EM, Aedo MV et al. Características de los pacientes con enfermedad crónica avanzada incluidos en un programa de cuidados paliativos domiciliario. Medicina Paliativa. 2017; 24 (4): 179-187.

2. San-Sebastián MK. Impacto de la atención paliativa en el grado de satisfacción percibida por los familiares del paciente en situación de final de vida en el área de Bizkaia [tesis inédita de doctorado]. Universidad del país Vasco. España. 2016.

3. Organización Mundial de la Salud. Cuidados Paliativos [internet]. 2018. Disponible en: https://www.who.int/es/ news-room/fact-sheets/detail/palliative-care

4. Organización Mundial de la Salud. Cáncer/Cuidados Paliativos [internet]. 2015. Disponible en: http://www.who. int/cancer/palliative/es/

5. Consejo de Salubridad Ceneral. Guía del manejo integral de cuidados paliativos [internet]. CSC. México. 2018. Disponible en: http://www.geriatria.salud.gob.mx/ descargas/publicaciones/Guia_cuidados_paliativos_ completo.pdf

6. Marrero MMDS. La evaluación de la calidad de vida en pacientes y familiares como indicadores de impacto de un programa de cuidados paliativos [tesis inédita de doctorado]. Universidad de Las Palmas de Gran Canaria. España. 2005.

7. Hernández-Bello E, Bueno-Lozano M, Salas-Ferrer P, Sanz-Armunia M. Percepción de la calidad de vida y satisfacción con la asistencia sanitaria recibida en un grupo de pacientes oncológicos terminales que reciben cuidados paliativos en un hospital de Zaragoza, España. Revista Colombiana de Cancerología. 2016; 20 (3): 110-115.

8. Traveso TG, Talavera AF, Sánchez AMB, Figuero LSB, Castrillón JLP. Grado de satisfacción e importancia de los cuidados durante la fase final de la vida. Medicina Paliativa. 2016; 23 (4): 79-182.
9. Velilla EDC, Jaramillo CMP. Calidad de vida y satisfacción en cuidados paliativos del HUM durante marzo y abril de 2018 [Tesis Inédita de Maestría]. Universidad del Rosario. Bogotá, Colombia. 2018.

10. Puente CP, Furlong LV, Romero FM, Rodríguez RM. Comunicación, calidad de viday satisfacción en pacientes de cuidados paliativos. Psicología y Salud. 2005; 15 (2): 195-206.

11. Turriziani A, Attanasio G, Scarcella F, Sangalli L, Scopa A, Genualdo A et al. The importance of measuring customer satisfaction in palliative care. Future Oncology. 2016; 12 (6): 807-813.

12. Urzúa A, Caqueo-Urízar A. Calidad de vida: Una revisión teórica del concepto. Terapia Psicológica. 2012; 30 (1): 61-71.

13. Guevara H, Domínguez A, Ortunio M, Padrón D, Cardozo R. Percepción de la calidad de vida desde los principios de la complejidad. Revista Cubana de Salud Pública. 2010; 36: 357-360.

14. Ullrich A, Ascherfeld L, Marx G, Bokemeyer C, Bergelt C, Oechsle K. Quality of life, psychological burden, needs, and satisfaction during specialized inpatient palliative care in family caregivers of advanced cancer patients. BMC Palliative Care. 2017; 16 (1). doi: 10.1186/s12904-017-0206-z.

15. Groenvold M, Petersen MA, Aaronson NK, Arraras JI, Blazeby JM, Bottomley A et al. The development of the EORTC QLQ-C15-PAL: a shortened questionnaire for cancer patients in palliative care. European Journal of Cancer. 2006:42(7):55-64.

16. Arraras JI, de la Vega FA, Asin G, Rico M, Zarandona U, Eito Cet al. The EORTC QLQ-C15-PAL questionnaire: validation study for Spanish bone metastases patients. Quality of Life Research. 2014;23(3):849-855.

17. Alawneh A, Yasin H, Khirfan G, Qayas BA, Ammar K, Rimawi D, Klepstad P. Psychometric properties of the Arabic version of EORTC QLQ-C15-PAL among cancer patients in Jordan. Supportive Care in Cancer. 2016;24(6):2455-2462.

18. Bredart A, Bottomley A, Blazeby JM, Conroy T, Coens C, D'haese $S$ et al. An international prospective study of the EORTC cancer in-patient satisfaction with care measure (EORTC IN-PATSAT32). European Journal of Cancer. 2005; 41 (14): 2120-2131.

19. Secretaría de Salud. Ley General de Salud en Materia de Investigación para la Salud. SECOB. 2014. Recuperado de: https://www.dof.gob.mx/nota_detalle.php?codigo=533916 2\&fecha $=02 / 04 / 2014$

20. Cómez MMN, Caycedo C, Aguillón M, Álvarez RAS. Calidad de vida y bienestar psicológico en pacientes adultos con enfermedad avanzada y en cuidado paliativo en Bogotá. Pensamiento psicológico. 2008;4(10):177-195.

21. Geidy LM. Satisfacción de pacientes con cáncer de pulmón avanzado incluidos en ensayos clínicos en villa clara, cuba. Año 2014. In convención salud 2015. 2015. 